\title{
Monitoria em laboratório de práticas de enfermagem: relato de experiência
}

\author{
Monitoring in laboratory of practices of nursing: report experience \\ Seguimiento de laboratorio de las prácticas de enfermería: relato de experiencia
}

\section{Resumo}

Este artigo objetiva relatar a experiência de estudantes de enfermagem como monitoras de um laboratório de práticas de enfermagem de uma universidade comunitária. Trata-se de um relato de experiência, realizado por estudantes de enfermagem, referente as ações desenvolvidas no período de março de 2018 a outubro de 2020, por meio da monitoria do laboratório de práticas de enfermagem, que está vinculado ao curso de graduação em Enfermagem de uma Universidade Comunitária. As experiências sustentam-se em quatro pilares: a necessidade constante de revisão da teoria para associá-la com as atividades práticas; o aprimoramento das relações de comunicação interpessoal entre a tríade monitor-estudante-docente; o desenvolvimento prático do exercício ético profissional; e aproximação do gerenciamento do laboratório com a prática de gerenciamento em enfermagem. As ações proporcionaram a potencialização da formação crítico-reflexiva, o compartilhar de experiências na construção do processo ensinoaprendizagem e o aprimoramento das habilidades técnicas-práticas-científicas das estudantes.

Palavras-chave: Estudantes de enfermagem; Educação em enfermagem; Aprendizagem; Laboratórios.

\begin{abstract}
This article objective report the experience of students of nursing as monitors in a laboratory practice nursing of a community university. It is na report experience, realized with students nursing, relative to the actions developed a from March 2018 to October 2020, through the monitoring of the nursing practices laboratory, which is linked to the undergraduate nursing course of a Community University. The experiences are based on four pillars: the constant needto revise the theory to associate it with practical activities; the improvement of interpersonal communication between the triad monitor-student-teacher; the practical development of exercise ethical professional; and approximation of management of the laboratory with the practice management in nursing. The actions provided the potentialization of formation of critical-reflective, the share of experiences in construction of the teaching-learning process and the enhancement of the technical-practical-scientific abilityof the students.
\end{abstract}

Keywords: Students nursing; Education nursing; Learning; Laboratories.

\section{Resumen}

Este artículo tiene como objetivo reportar la experiencia de estudiantes de enfermería como monitores en un laboratorio de práctica de enfermería en una universidad comunitaria. Se trata de un relato de experiencia, realizado por estudiantes de enfermería, respecto a las acciones desarrolladas desde marzo de 2018 a octubre de 2020, a través del seguimiento del laboratorio de prácticas de enfermería, que está vinculado al curso de graduación en enfermería Universidad Comunitaria. Las experiencias se basan en cuatro pilares: la constante necesidad de revisar la teoría para asociarla con actividades prácticas; la mejora de las relaciones de comunicación interpersonal entre la tríada monitorestudiante-profesor; el desarrollo práctico del ejercicio ético profesional; y aproximación de la gestión de laboratorio con la práctica de gestión de enfermería. Las acciones propiciaron la potenciación de la formación crítico-reflexiva, el compartir de experiencias en la construcción del proceso de enseñanza-aprendizaje y la mejora de las competencias técnico-prácticas-científicas de los estudiantes.

Palabras clave: Estudiantes de enfermería; Educación en enfermería; Aprendizaje; Laboratorios. 


\section{Introdução}

A formação profissional atualiza-se constantemente devido aos cenários educativos, laborais, políticos, econômicos e sociais que acompanham as transformações globais. Estes cenários, vivenciam transições demográficas, revoluções tecnológicas, educacionais, culturais e epistemológicas, que embasam e incentivam à transformação, adaptação e inovação das instituições de ensino. Diante disso, a formação em saúde, especialmente em enfermagem, necessita formar enfermeiros capazes de liderar equipes detentoras de múltiplos saberes e práticas, com uma visão global e interconectada com os avanços tecnológicos e culturais (Frota, et al., 2020).

A formação do enfermeiro alinha-se com as Diretrizes Curriculares Nacionais do Curso de Graduação em Enfermagem (DCENF), que é pautada em uma formação generalista, humanista, crítica e reflexiva. Tende a abordar a qualificação do profissional para o exercício da profissão, com competência para atuar em situações-problema de saúdedoença, munido de responsabilidade e compromisso com a sociedade, sendo promotor de saúde ao ser humano em sua integralidade (Brasil, 2001).

Além disso, é fundamental que os Cursos de Graduação em Enfermagem desenvolvam atividades que associem o ensino-aprendizagem aos cenários de atuação profissional. Esse processo educativo deve estar orientado pela interdisciplinaridade do conhecimento, a integralidade da formação e a interprofissionalidade das práticas e do trabalho em saúde. E essa articulação de conteúdos teóricos e práticos, são fundamentais para a construção de competências e habilidades e podem ser desenvolvidas em laboratórios de simulação ou de práticas de enfermagem (Brasil, 2018).

Assim, o laboratório de práticas de enfermagem é um espaço voltado para os estudos, propicia aos estudantes e professores desenvolverem as aulas práticas, e auxilia o estudante de enfermagem a desenvolver as habilidades técnicas, conciliando-as com as atividades teórico/práticas. Tal dinâmica resulta em melhorias no desempenho educacional ao instigar a criatividade e o raciocínio crítico do aluno (Brito, Rozendo \& Melo).

O laboratório de enfermagem vem com uma perspectiva para além do desenvolvimento de apenas habilidades técnicas. Também, é um espaço voltado para aproximar o estudante da realidade de trabalho do enfermeiro, por meio de simulações de resoluções de problemas e organização e planejamento de ações, tempo e materiais. Contribui para o estudante sentir-se responsável pelos seus atos e respeitar o ser humano na sua integralidade, de forma que quando este se encontrar diante do paciente, ele possa sentir-se seguro com a realização dos procedimentos, e desenvolver uma postura profissional baseada em princípios éticos-morais (Brito, Rozendo \& Sobral, 2018).

Soma-se a isto, o fato de que laboratório de enfermagem possibilita aos estudantes vivenciarem a experiência de atuarem como monitores deste espaço. A monitoria acadêmica é compreendida como uma forma de apoio ao ensino/aprendizagem, em que o monitor/estudante contribui no processo de apreensão do conhecimento e no aprimoramento das técnicas de enfermagem dos demais estudantes (Burgos, et al., 2019).

Nessa perspectiva, a monitoria acadêmica tem se mostrado como uma importante estratégia pedagógica para o processo de ensino aprendizagem. Assim, por meio da Lei $n^{\circ}$ 9.394/1996, no seu artigo 84, foi regulamentado e estabelecidoque os estudantes de graduação do ensino superior poderão ser inseridos em atividades de ensino e pesquisa pelas respectivas instituições de ensino, desenvolvendo funções de monitoria, desde que esteja em consonância com seu rendimento e com o projeto político pedagógico dos cursos (Brasil, 1996).

Diante do exposto, despertou-se o interesse de relatar a experiência de estudantes de enfermagem como monitoras de um laboratório de práticas de enfermagem de uma universidade comunitária. Deste modo, o presente estudo tem como objetivo relatar a experiência de estudantes de enfermagem como monitoras de um laboratório de práticas de enfermagem. 


\section{Metodologia}

Trata-se de um relato de experiência, referente as ações desenvolvidas por estudantes de enfermagem como monitoras de um laboratório de práticas de enfermagem.

As ações ocorreram no período de março de 2018 a outubro de 2020, por meio da monitoria do laboratório de práticas de enfermagem, que está vinculado ao curso de graduação em Enfermagem de uma Universidade Comunitária localizada na região Centro-Oeste do Rio Grande do Sul (RS), Brasil. As atividades de monitoria foram desenvolvidas por três estudantes concluintes do curso de graduação em Enfermagem entre 2018 e 2020, sob a supervisão de docente do curso e responsável pelo laboratório.

Dentre os cursos de graduação ofertados pela universidade, está o curso de Enfermagem que é composto por dez semestres com aulas teórico-práticas e estágio curricular supervisionado. O curso de Enfermagem, possui em anexo o laboratório de práticas de Enfermagem, o qual é um espaço voltado para o exercício de técnicas e procedimentos para os estudantes da área do conhecimento de Ciências da Saúde, priorizando o aprimoramento de habilidades e destrezas para o exercício da profissão. Objetiva desenvolver os conhecimentos teóricos, oportunizando a simulação de situações para a realidade do trabalho profissional (Uri, 2020a).

E o período das atividades de monitoria é de segunda a sexta-feira, com uma carga horaria semanal de 20 horas. O monitor assume responsabilidades de coordenar o espaço, mantendo o ambiente limpo e organizado, zelando e preparando o ambiente para dispor dos materiais para as aulas/atividades previamente agendadas. Ademais, o monitor auxilia os docentes durante o transcorrer das aulas práticas, bem como salienta a importância de cumprir os regulamentos, normas e rotinas deste ambiente, além de registrar e controlar o cronograma referente as aulas práticas agendadas e o livro de controle de entrada e saída de materiais do laboratório (Uri, 2020a).

Ressalta-se ainda, que as atividades de gestão do laboratório de prática de Enfermagem são de responsabilidade de um docente do curso de enfermagem, juntamente com o estudante monitor.

\section{Resultados}

O relato de experiência das estudantes de enfermagem como monitoras de um laboratório de práticas de enfermagem sustenta-se em quatro pilares que contribuíram para o fortalecimento da formação em enfermagem, como: a necessidade constantemente de revisão da teoria para associá-la com as atividades práticas; o aprimoramento das relações de comunicação interpessoal entre a tríade monitor-estudante-docente; o desenvolvimento prático do exercício ético profissional; e aproximação do gerenciamento e gestão do laboratório com a prática de gerenciamento em enfermagem.

O primeiro pilar trata-se da relevância de estar constantemente estudando os conteúdos teóricos para associá-los com as atividades práticas. Este fato está relacionado a necessidade, observada pelas estudantes monitoras, de se manterem atualizadas sobre o conhecimento teórico-científico, e reconhecer a importância de previamente revisar os conteúdos, para que assim pudessem desenvolver com segurança e qualidade as habilidades técnicas, tanto para demonstração de procedimentos, quanto para observar e auxiliar outros estudantes.

O segundo pilar, refere-se ao processo de aprimoramento das relações de comunicação interpessoal entre a tríade monitor-estudante-docente. Esta circunstância dá-se pela importância de haver uma comunicação compreensível e eficaz, devido a relação interpessoal se fazer presente em diversos momentos da prática da monitoria. Esses momentos, perpassam desde os agendamentos de aulas práticas por parte do docente e/ou estudantes, como também em auxilia-los durante essas aulas práticas e até mesmo para a organização e solicitação de compra de materiais utilizados no laboratório. Destaca-se ainda, que por meio da comunicação ocorrem trocas de conhecimentos e experiências entre os envolvidos da tríade. 
O terceiro pilar apresentou-se para as monitoras como uma oportunidade de desenvolvimento prático do exercício ético profissional, pela necessidade de a monitora adotar uma postura ética, responsável e sigilosa frente ao processo de organização e andamento das questões burocráticas que estão envoltas neste espaço pedagógico. Destaca-se que o exercício ético profissional perpassa todos os seguimentos da monitoria, faz-se necessário ser coerente com as ações desde o agendamento do laboratório até o portar-se durante as simulações das aulas práticas.

Ainda, possibilita que os cuidados prestados na atuação da profissão possam ser praticados dentro do laboratório, a fim de reduzir possíveis riscos e danos à saúde dos pacientes. Considera-se que o monitor também é um estudante, e tais práticas nos manequins de simulação propiciam ao monitor uma qualificação ao prestar a assistência, como também não conduzir procedimentos dolorosos em pacientes somente para incrementar as suas habilidades. Assim, a monitoria fortaleceu a relação entre o conhecimento teórico-prático para o desempenho de uma prática de reverência no âmbito da profissão, fundamentada nos direitos e deveres da enfermagem, e baseada em princípios éticos-morais.

O quarto pilar, trata-se da aproximação do gerenciamento e gestão do laboratório com a prática de gerenciamento em enfermagem, e nessa interface a monitoria requer do estudante compromisso de manter o espaço pedagógico íntegro e apto para as aulas práticas, sendo o monitor o principal responsável por este espaço e pelo andamento satisfatório das atividades. Dessa forma, cabe a monitora manter o laboratório organizado, com um cronograma para guiar os agendamentos das aulas práticas a serem exercidas tanto pelos docentes quanto para os demais estudantes, a fim de não cruzar a utilização do espaço durante o mesmo horário.

Ainda nesse contexto, compete a monitora controlar a entrada e saída de materiais, tendo em vista que alguns materiais também são utilizados fora deste espaço pedagógico. Ressalta-se que os materiais que saem do laboratório são registrados em uma agenda específica. Além disso, a monitora realiza o dimensionamento de materiais, por meio do controle de uso e a solicitação de novos materiais, sempre atentando para manter a disponibilidade dos mesmos durante as práticas.

Em suma, incumbe também ao estudante monitor, orientar os frequentadores do espaço quanto ao uso correto dos Equipamentos de Proteção Individual (EPI) com o propósito de reduzir os riscos ameaçadores a saúde e garantir a segurança dos mesmos durante as aulas. Ainda, a monitora instrui sobre o descarte adequado dos resíduos sólidos, para evitar possíveis contaminações e reduzir custos tanto para o espaço pedagógico como também para futuramente nos serviços de assistência à saúde.

\section{Discussão}

A formação profissional do curso de graduação em Enfermagem da presente Universidade Comunitária, está embasada no perfil generalista, qualificada para o exercício da enfermagem, tendo como fundamento uma perspectiva humanista, crítica, reflexiva e ética, com capacidade e habilidade de intervir sobre problemas/situações de saúde/doença do ser humano nos diversos cenários de serviços à saúde. Além disso, o curso proporciona aos estudantes a independência na atuação profissional e na colaboração entre equipes interdisciplinares e multiprofissionais durante o processo de saúde/doença (Uri, 2020b).

Assim, para o desenvolvimento profissional em enfermagem ser eficaz, faz-se necessário estar em constante revisão teórica para associar com as atividades práticas. Isso, vem ao encontro do primeiro pilar exposto, como também aos princípios da formação em enfermagem que requer o rigor técnico-científico, para que as ações em saúde sejam práticas baseadas em evidências. Dessa maneira, a formação do enfermeiro deve estar voltada para uma formação permanente, humanística e técnico-científica e o mesmo sentir-se como sujeito do seu processo formativo e facilitador do processo de desenvolvimento dos profissionais que compõem a equipe de enfermagem (Brasil, 2018). 
No que tange ao segundo pilar, as relações de comunicação interpessoal em enfermagem têm-se apresentado como uma excelente estratégia no meio formativo para impulsionar o pensamento crítico, a fim de problematizar e respeitar as diferenças e acolher as diversidades. Considera-se ainda, que a presença dos múltiplos sujeitos auxilia para que essa relação ocorra numa dimensão de respeito e responsabilidade entre os envolvidos (Brito, Rozendo \& Melo, 2018).

Além do mais, a simulação clínica em laboratório contribuí de forma significativa para o desenvolvimento de competências da comunicação aprimorando a habilidade de comunicação e propicia aos estudantes a vivência de situações que exigem tomada de decisão do enfermeiro nos cenários de cuidado em saúde e enfermagem cuja abordagem por meio de aulas teóricas não têm a mesma eficácia (Santos, et al., 2019).

Dessa forma, entende-se que a ação de cuidar implica em estabelecer uma relação interpessoal, na qual é importante singularizar a experiência de cada indivíduo. Assim, a formação de enfermeiros necessita de um olhar crítico voltado para a construção de sujeitos éticos, solidários e emancipados. Para que isso ocorra, os docentes devem buscar possibilidades de discussão e de enfrentamento de tomada de decisões que contribuirão para um cuidado de enfermagem coerente com as reais necessidades dos sujeitos. Sendo assim, o laboratório de enfermagem propicia assimilação e apreensão dos conhecimentos já estabelecidos, e auxilia caminhar em direção a um processo de construção ativa de novas compreensões da realidade e (re)invenção dos saberes e práticas (Brito, Rozendo \& Sobral, 2018).

Quanto ao terceiro pilar descrito, a Enfermagem necessita atuar com autonomia e em consonância com os preceitos éticos e legais, técnico-científico e teórico-filosófico, assegurando exercer as atividades com competência para promoção do ser humano na sua integralidade; garantindo a universalidade de acesso, integralidade da assistência, resolutividade e preservação da autonomia das pessoas. Tais aspectos, vem ao encontro do Art. $6^{\circ}$ da Resolução n ${ }^{0}$ 564/2017 do COFEN, corroborando que aprimorar os conhecimentos técnicos-científicos, éticos-políticos, socioeducativos, históricos e culturais darão sustentação a prática profissional do enfermeiro (Cofen, 2017).

Ressalta-se que as questões éticas precisam ser enfatizadas, assim como, a assistência à saúde requer uma reflexão dos profissionais. O profissional necessita atuar de maneira ética e humanizada, e ser capaz de administrar todas as interfaces do cuidado. A equipe de saúde, deve compreender esta postura e focar a assistência do indivíduo de modo a resgatar sua dignidade, transmitir-lhe segurança e confiança. O enfermeiro, precisa estar preparado para a tomada de decisão em situações de complexidade, esta visão, é para além da prática assistencial, é para o enfrentamento e resolução dos conflitos éticos (Santos $\&$ Baleeiro, 2012).

Desse modo, o cuidado de Enfermagem fundamenta-se no conhecimento próprio da profissão e nas ciências humanas, sociais e aplicadas, possibilitando executar a prática profissional com ênfase no assistir, gerenciar, ensinar, educar e pesquisar. Neste sentido, o processo de monitoria também possibilita ao estudante obter uma visão ampla de gerenciamento e gestão, o que está em conformidade com o quarto pilar explanado e com a Lei 7.498/1986 que dispõe sobre o Exercício da Enfermagem. Destaca-se ainda, que no seu Art. 11, incumbe como atribuição do profissional enfermeiro o planejamento, organização, coordenação, execução e avaliação dos serviços da assistência de Enfermagem (Cofen, 1986).

A Enfermagem desenvolve ações de prevenção, promoção, proteção e reabilitação da saúde tanto a nível individual quanto coletivo. Além disso, a Enfermagem está atenta aos resíduos geradores de suas atividades práticas, a fim de minimizar os riscos de infecções cruzadas e ambientais à saúde tanto dos profissionais quanto aos clientes. Dessa forma, considera-se que o enfermeiro é um profissional apto para desempenhar a função de gerenciar os resíduos dos serviços de saúde, e assim, é fundamental que esteja conscientizado e saiba classifica-lo, manuseá-lo e gerencia-lo corretamente no decorrer das atividades práticas (Nascimento \& Maia, 2010).

Ainda nesse meio gerencial, cabe a enfermagem visar a utilização correta dos EPIs, por meio da observação se o indivíduo faz o uso adequado dos equipamentos, como também esclarecendo dúvidas e orientando-o. Logo, torna-se 
primordial que no decorrer do processo formativo ocorra a fomentação de discussões e suscitação de reflexões acerca do uso correto dos EPIs, a fim de prevenir acidentes de origem física, química ou biológica, considerado ameaçadores para si e para o coletivo (Ferreira \& Nascimento, 2017).

Em vista disso, entende-se que a formação em Enfermagem necessita estar pautada no gerenciamento e administração tanto da força de trabalho quanto dos recursos físicos materiais e de informação, portanto, o enfermeiro está preparado para o desenvolvimento de ações empreendedoras de gestão e liderança da equipe de saúde. Além de todos esses aspectos, a formação também permite focar atenção à saúde, tomada de decisão, comunicação e educação permanente (Brasil, 2001).

\section{Conclusão}

A experiência como monitoras de um laboratório de práticas de enfermagem contribuiu significativamente para potencializar a formação crítico-reflexiva, o compartilhar de experiências na construção do processo ensino-aprendizagem e para o aprimoramento das habilidades técnicas-práticas-cientificas das estudantes. Além disso, destaca-se que o laboratório é para além de um espaço físico, mas sim um espaço pedagógico, que colabora construtivamente para a formação em Enfermagem.

\section{Referências}

Brasil, Ministério da Educação. (2001). Conselho Nacional de Educação (CNE). Parecer CNE/CES $n^{\circ} 3$ de 7 de novembro de 2001. Institui Diretrizes Curriculares Nacionais dos Cursos de Graduação em Enfermagem. Diário Oficial da União.

Brasil, Ministério da Educação. (1996). Lei $n^{\circ}$ 9.394, de 20 de dezembro de 1996. Estabelece as diretrizes e bases da educação nacional. Diário Oficial da União.

Brasil, Ministério da Saúde. (2018) Conselho Nacional de Saúde. Resolução $n^{\circ}$ 573, de 31 de janeiro de 2018. Diário Oficial da União, Brasília, DF.

Brito F. M. M., Rozendo C. A., \& Melo P. O. C. (2018). Laboratório de enfermagem e a formação crítica de enfermeiros: aproximações e distanciamentos. Rev. Bras. Enferm. 71(4): 1500-1506.

Brito F. M. M., Rozendo C. A., \& Sobral J. P. C. P. (2018). O laboratório de enfermagem e a formação crítica do enfermeiro: uma reflexão. Enfermagem em Foco. 9(1):36-40.

Burgos C., Baricati C., Martins J., Scholze A., Galdino M., \& Karino M. (2019). Monitoria acadêmica na percepção dos estudantes de enfermagem. Rev. Enferm. UFSM. 9(37):1-14

Cofen, Conselho Federal de Enfermagem. (1986). Lei $n^{\circ} 7.498 / 86$, de 25 de junho de 1986. Dispõe sobre a regulamentação do exercício da enfermagem, e dá outras providências. Brasília.

Cofen, Conselho Federal de Enfermagem. (2017). Resolução COFEN no 564/2017, de 6 de novembro de 2017.

Ferreira R. G. S., \& Nascimento J. L. (2017). Interface educação continuada/enfermagem do trabalho: otimizando a usabilidade dos EPI’S em clínica médica. Revista Recien. 7(20):105-114.

Frota M. A., Wermelinger M. C. M. W., Vieira L. J. E. S., Ximenes N. F. R. G., Queiroz R. S. M., \& Amorim R. F. (2020). Mapeando a formação do enfermeiro no Brasil: desafios para atuação em cenários complexos e globalizados. Ciênc. saúde coletiva. 25(1): 25-35.

Nascimento E. B., \& Maia L. F. S. (2010). O enfermeiro no gerenciamento de resíduos dos serviços de saúde. Revista Recien. 1(1):27-32.

Santos C. J., \& Baleeiro J. F. (2012). A visão do enfermeiro sobre o ponto de vista ético do paciente crítico em UTI. Revista Recien. 2(4):5-9

Santos J. L. G., Copelli F. H. S., Balsanelli A. P., Sarat C. N. F., Menegaz J. C., Trotte L. A. C., et al. (2019). Interpersonal communication competence among nursing students. Rev. Latino-Am. Enfermagem. 27: e3207.

Uri, Universidade Regional Integrada do Alto Uruguai e das Missões. (2020). Manual de Normas e Rotinas do Laboratório de Enfermagem.

Uri, Universidade Regional Integrada do Alto Uruguai e das Missões. (2020) Resolução $n^{o}$ 2752/CUN/2020. Dispõe sobre Projeto Pedagógico do Curso de Enfermagem da URI. Santiago, RS.b 